\title{
Clinical Analysis of Central Pontine Myelinolysis after Liver Transplantation
}

\author{
Zhixin Wang1, Ruiyao Zhang1, Kai Qu'1, Yanyan Zhou', Wei Chen1, Xinsen Xu', \\ Fandi Meng', Jichao Wei ${ }^{1}$, Minghui Tai ${ }^{1}$, Jingyao Zhang', Lingqiang Zhang1, \\ Yuelang Zhang', Chang Liu ${ }^{1}$ \\ ${ }^{1}$ Department of Hepatobiliary Surgery, The First Affiliated Hospital, Medical School of Xi'an Jiaotong University, \\ Xi'an, China \\ ${ }^{2}$ Department of Radiology, The First Affiliated Hospital, Medical School of Xi'an Jiaotong University, Xi'an, China \\ ${ }^{3}$ Department of neurology, The First Affiliated Hospital, Medical School of Xi'an Jiaotong University, Xi'an, China \\ Email: zhixin wang001@sina.com
}

Received 8 April 2014; revised 18 May 2014; accepted 7 July 2014

Copyright (C) 2014 by authors and OALib.

This work is licensed under the Creative Commons Attribution International License (CC BY). http://creativecommons.org/licenses/by/4.0/

(c) (i) Open Access

\begin{abstract}
Objective: To discuss the etiology, diagnosis and treatment of central pontine myelinolysis (CPM) by analyzing 4 cases of our center and 26 cases reported by others in our country. Methods: We analyzed the clinical feature, diagnosis and treatment of 4 CPM cases and then compared them with 26 CPM cases reported between 2000 and 2010. Results: The average age of our 4 cases (3 men and 1 woman) was 47.5 years old. All cases were diagnosed by MRI scan. The patients all had been diagnosed as liver cirrhosis and most of the CPM related symptoms were observed within 2 weeks after surgery, including neural and psychic symptoms and physical impairment. Rapid correction of hyponatremia during perioperative period and high blood concentration of FK506 may be the main causes. All cases received etiological and symptomatic combination treatment and were continued with neural function training after hospital discharge. A long-term follow-up (29.5 \pm 15.0 months) showed an improvement of neural function to different degrees. Conclusion: CPM after liver transplantation is a severe complication, of which the etiology and pathogenesis were not clear. MRI scan is the main method for confirmative diagnosis. Etiological prevention and timely treatment may improve the prognosis.
\end{abstract}

\section{Keywords}

Liver Transplantation, Central Pontine Myelinolysis, Clinical Analysis

Subject Areas: Gastroenterology \& Hepatology, Surgery \& Surgical Specialties

\section{Introduction}

Central pontine myelinolysis (CPM) after liver transplantation was first reported by Starzl in 1987, of which the

How to cite this paper: Wang, Z.X., Zhang, R.Y., Qu, K., Zhou, Y.Y., Chen, W., Xu, X.S., Meng, F.D., Wei, J.C., Tai, M.H., Zhang, J.Y., Zhang, L.Q., Zhang, Y.L. and Liu, C. (2014) Clinical Analysis of Central Pontine Myelinolysis after Liver Transplantation. Open Access Library Journal, 1: e759. http://dx.doi.org/10.4236/oalib.1100759 
main pathological characteristic is myelinolysis in central pontine caused by various reasons after liver transplantation, and the typical clinical characteristics are limb paralysis, pseudobulbar palsy, locked-in syndrome, etc. However, the pathogenesis of CPM has not been clearly identified so far. In this paper, we studied the data of 4 CPM cases in our center and analyzed the clinical features and possible causes of CPM after liver transplantation.

\section{Material and Method}

\subsection{Clinical Data}

Between November 2000 and December 2010, consecutive orthotopic liver transplantation (OLT) was performed on 184 patients in our center. 4 of them were diagnosed as central pontine myelinolysis (CPM) after the operation, and their medical records were retrospectively investigated. Then we retrieved all the literatures from the network database like CNKI, and WANFANG DATA with "central pontine myelinolysis after liver transplantation" as keywords. After removing irrelevant, internal journal and review literature, selecting literatures reported cases in accord with the diagnosis of "central pontine myelinolysis after liver transplantation", further removing those that lack important clinical data, we finally got 15 effective literatures [1]-[15]. All data were based on the reported number.

\subsection{Statistical Analysis}

Set up data base with information of both cases and articles. Measurement data was expressed as mean \pm SD and enumeration data was expressed as a percentage. Make Use of SPSS 19.0 software for data statistics and analysis.

\section{Result}

\subsection{General Data}

Since 2000, consecutive orthotropic liver transplantation (OLT) was performed on nearly 200 cases in our center, 4 of which were diagnosed as CPM after liver transplantation. Of the 4 patients, 3 were male and 1 was female, and their average age was $47.5 \pm 10.5$ years. The primary diseases were hepatic cirrhosis in 3 cases and chronic severe hepatitis in 1 case. Among the 4 patients, 1 patient showed hepatic encephalopathy before the operation, and 2 patients had diabetes. All 4 cases in our center received classic orthotropic liver transplantation (OLT) (Table 1). By retrieving the previous officially published literatures, we got 15 literatures available, including 12 Chinese literatures, 2 English literatures and 1 master thesis. Among the 26 cases from the literatures, 23 cases (88.5\%) were male and 3 cases (11.5\%) were female. Their average age was $47.15 \pm 8.91$ years old. The primary diseases were severe hepatitis in 7 cases, hepatic B cirrhosis in 16 cases, liver carcinoma, drug liver failure, IgA nephropathy, and hepatolenticular degeneration in 1 case, respectively.

\subsection{Postoperative CPM}

The symptoms of CPM appeared after the operation at an average time of $9.0 \pm 6.0$ days, of which the main clinic features were nerve, mental symptoms and consciousness change (Table 2). All of these 4 cases had MRI scanned, showing a decreased signal intensity of T1-weighted images in the pontine without space occupying sign and an increased signal intensity of T2-weighted images in central pontine (Figure 1). All 26 cases from the literatures showed many symptoms in a sequence as below: paralysis, disturbance of consciousness, pseudobulbar palsy, epilepsia, Locked-in syndrome, mental disorders and oculomotor disorders (Figure 2).

\subsection{Blood Sodium Fluctuations and Hepatic Encephalopathy}

The 4 cases in our center all showed a great increase (more than $12 \mathrm{mmol} / \mathrm{L}$ ) in blood sodium concentration within the 24-hour perioperative period (Table 3). Among them, case 1, 2 and 3 showed psychiatric symptoms to different extents. 26 cases reported in articles suffered a treatment of a rapid correction of hyponatremia (more than $12 \mathrm{mmol} / \mathrm{L}$ ) in 24 hours around the operation, too, and also had preoperative hepatic encephalopathy (Table 4). 
Table 1. General condition, primary disease and surgical approach of 4 cases in our hopital.

\begin{tabular}{cccccc}
\hline case & Age (y) & Gender & Primary disease & $\begin{array}{c}\text { Preoperative hepatic } \\
\text { encephalopathy }\end{array}$ & Operation manner \\
\hline$\# 1$ & 68 & $\mathrm{~F}$ & Hepatitis cirrhosis (HBV) Diabetes type $\Pi$ & $\mathrm{N}$ & OLT \\
$\# 2$ & 45 & $\mathrm{M}$ & Hepatitis cirrhosis (HBV) Diabetes type $\Pi$ & $\mathrm{Y}$ & OLT \\
$\# 3$ & 29 & $\mathrm{M}$ & Chronic severe hepatitis & $\mathrm{N}$ & OLT \\
$\# 4$ & 48 & $\mathrm{M}$ & Hepatitis cirrhosis (HBV) & $\mathrm{N}$ & OLT \\
\hline
\end{tabular}

Table 2. Clinical manifestation and prognosis of 4 cases in our hopital.

\begin{tabular}{|c|c|c|c|c|c|c|}
\hline \multirow{2}{*}{ case } & \multirow{2}{*}{$\begin{array}{l}\text { Onset time } \\
\text { (d) }\end{array}$} & \multirow{2}{*}{ Cardinal symptom } & \multicolumn{2}{|c|}{ Complication } & \multirow{2}{*}{ Outcome } & \multirow{2}{*}{$\begin{array}{l}\text { Follow-up } \\
\text { time (m) }\end{array}$} \\
\hline & & & Infection & ARF & & \\
\hline \#1 & 12 & Dysarthria, double paralysis of the lower limbs & Y(lung) & $\mathrm{N}$ & Cured & 40 \\
\hline \#2 & 5 & $\begin{array}{c}\text { Confusion, speech increase, dysphoria, lack of logical thinking, } \\
\text { difficulty swallowing, motor aphasia }\end{array}$ & $\mathrm{N}$ & $\mathrm{N}$ & Cured & 49 \\
\hline \#3 & 5 & Emotional irritability, consciousness, hemiparesis, dysarthria, motor aphasia & $\mathrm{N}$ & $\mathrm{N}$ & Cured & 26 \\
\hline \#4 & 11 & Mental symptoms, disturbance of consciousness, coma & Y(lung) & $\mathrm{Y}$ & Cured & 3 \\
\hline
\end{tabular}

Table 3. Concentration of serum sodium and immunosuppressive agents of 4 cases in our hopital.

\begin{tabular}{cccccc} 
Case & $\begin{array}{c}\text { Preoperative } \\
{[\mathrm{Na}+] \mathrm{a}(\mathrm{mmol} / \mathrm{L})}\end{array}$ & $\begin{array}{c}\text { Postoperative } \\
{[\mathrm{Na}+] \mathrm{b}(\mathrm{mmol} / \mathrm{L})}\end{array}$ & $\Delta[\mathrm{Na}+](\mathrm{mmol} / \mathrm{L})$ & Immunosuppressor & High drug concentration c \\
\hline$\# 1$ & 123.9 & 142 & 18.1 & FK506 & $\mathrm{Y}$ \\
$\# 2$ & 112 & 141.1 & 19.1 & FK506 & $\mathrm{N}$ \\
$\# 3$ & 126.4 & 146 & 19.6 & FK506 & $\mathrm{Y}$ \\
$\# 4$ & 136.8 & 151 & 14.2 & FK506 & $\mathrm{Y}$ \\
\hline
\end{tabular}

a. preoperative $24 \mathrm{~h}[\mathrm{Na}+$, b. postoperative $24 \mathrm{~h}[\mathrm{Na}+]$, c. over $20 \mathrm{ng} / \mathrm{ml}$ at least once before the onset of CPM.

Table 4. Relationship between symptom time and rapid correction of hyponatremia, preoperative hepatic encephalopathy.

\begin{tabular}{|c|c|c|c|c|}
\hline Factor & & N (\%) & Onset time (d) & $P$ value \\
\hline \multirow{2}{*}{$\begin{array}{l}\text { Rapid correction of } \\
\text { hyponatremia }\end{array}$} & $\mathrm{Y}$ & $22(84.6)$ & $8.45 \pm 5.20$ & \multirow{2}{*}{0.021} \\
\hline & $\mathrm{N}$ & $4(15.4)$ & $11.50 \pm 5.20$ & \\
\hline \multirow{2}{*}{$\begin{array}{l}\text { Preoperative hepatic } \\
\text { encephalopathy }\end{array}$} & $\mathrm{Y}$ & $10(38.5)$ & $6.90 \pm 4.30$ & \multirow{2}{*}{0.001} \\
\hline & $\mathrm{N}$ & $16(61.5)$ & $10.19 \pm 5.36$ & \\
\hline
\end{tabular}
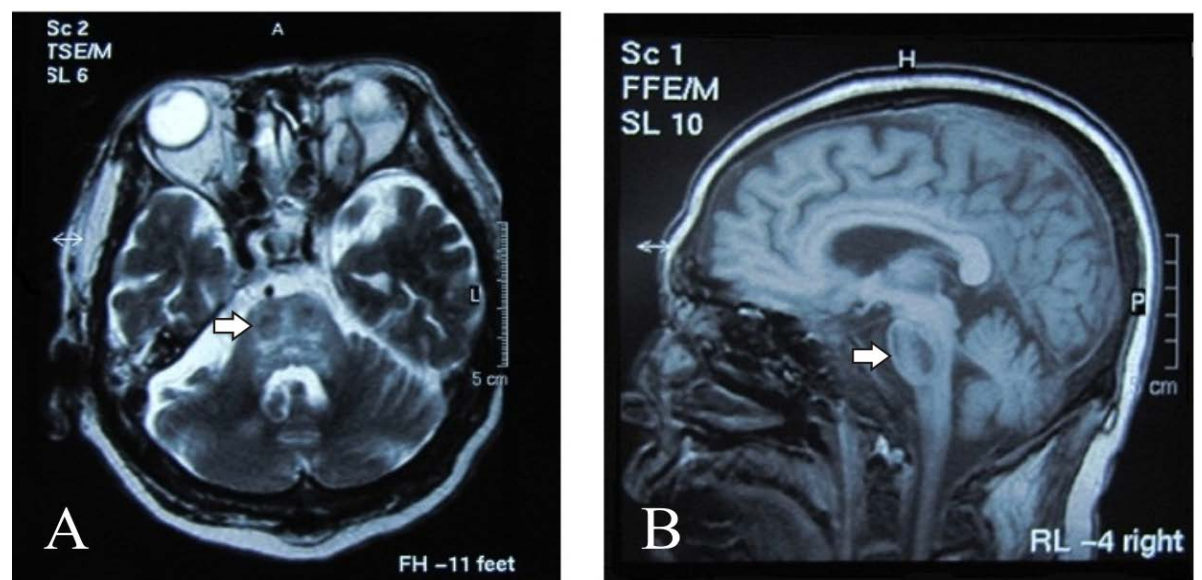

Figure 1. Skull MRI of case 4 (A: horizontal T2 scanning, B: sagittal T1 scanning). The arrow indicate lesion in central pontine, characteristicof which is a hypointensity signal of T1weighted images in the pontine without space occupying sign and increased signal intensity of T2-weighted images in central pontine. 


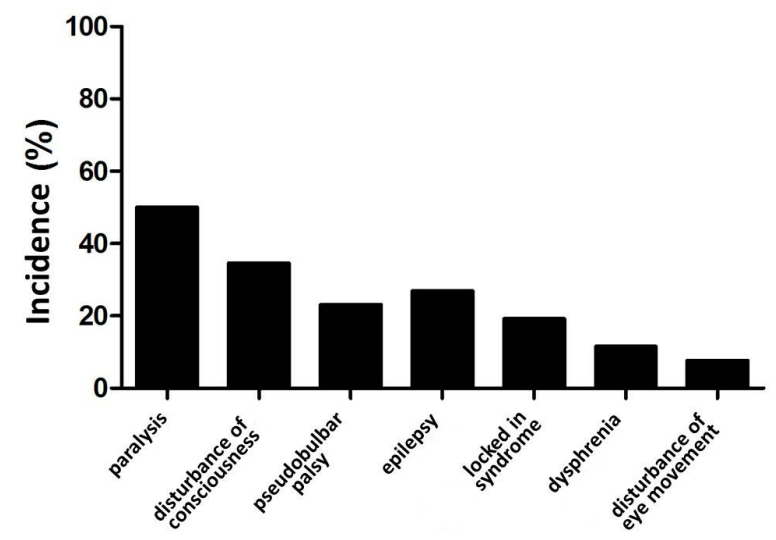

Figure 2. Clinical symptoms incidence of CPM reported in literature.

\subsection{Concentration of Immunosuppressive Drugs in Blood}

4 cases in our center took immunosuppressive drugs (FK506) after the operation in dose of $0.10-0.15 \mathrm{mg} / \mathrm{kg} / \mathrm{d}$, and were detected the blood concentration of FK506 every other day. 3 of them appeared a high level of FK506 concentration exceeding $20 \mathrm{ng} / \mathrm{ml}$ at least for one time (Table 3). 26 cases from the literatures have no detailed data of the blood concentration of immunosuppressive drugs.

\subsection{Treatment and Prognosis}

The 4 cases in our center received a comprehensive therapy, including nerve nutrition and brain function improvement, etc. Meanwhile, the liver function, kidney function, electrolyte and blood concentration of immunosuppressive drugs of the patients were monitored carefully. Among these 4 cases, both cases 1 and 4 suffered pulmonary infection and received anti-infection treatment; case 4 also suffered acute kidney failure and received continuous renal replacement therapy (CRRT). All of them were discharged and recovered to different degrees within an average time of $29.5 \pm 15.0$ months' follow up.

Among 26 cases reported in literatures, 17 (65.4\%) had concurrent infection, and for single factor regression analysis with clinical outcomes, the difference was statistically significant $(P<0.05) .11(42.3 \%)$ had concurrent kidney failure, and for single factor regression analysis with clinical outcomes, the difference was statistically significant $(P<0.05) .15(57.7 \%)$ cases were dead before discharge.

\section{Discussion}

As one of the leading death causes after liver transplantation [16] [17], CPM has aroused a wide awareness. Although the incidences are different in different countries according to various transplant centers' reports, the total incidence of CPM after liver transplantation lies between $5 \%$ and $10 \%$, which is much higher than that of the general population $(0.16 \%-5.8 \%)$.

\subsection{Etiology and Incentive}

As little known of the exact etiology and effective treatment of CPM, its prevention seems to be particularly important, and the present study shows that the etiology may but not limited to as follows.

\subsection{Rapid Correction of Hyponatremia and Preoperative Hepatic Encephalopathy}

Rapid correction of hyponatremia is one of the most widely accepted incentive factors of CPM, and some researchers have successfully made CPM animal models by correcting the hyponatremia rapidly [18]. The reasons of serum sodium concentration fluctuation for preoperative liver transplantation cases contain: a). As patients with end-stage liver diseases have poor ability to degrade hormone, the serum concentration of antidiuretic hormone and aldosterone are relatively high. Meanwhile, some patients with renal insufficiency were given 
long-term diuretic treatment, so almost all preoperative cases had low serum sodium, even chronic and intractable hyponatremia. b). Massive transfusion of plasma, albumin, red blood cells and $5 \%$ sodium bicarbonate during the operation can increase the sodium concentration within a short time [19]. Besides, as a result of the donor liver's normal ability to degrade antidiuretic hormone and aldosterone, a lot of water and relatively small amount of sodium excreted and as a result the concentration of serum sodium increased further. The great fluctuation of serum sodium levels in a short time can cause excessive fluctuations of plasma osmolality, endothelial injury, intercellular tight junctions open up, cross-vascular transshipment increases and large amounts of myelin toxic substances release, all contributing to the occurrence risk of CPM [20]. Abbasoglu et al. [21] regarded preoperative hepatic encephalopathy as another risk factor of CPM, and other scholars believed that the lack of brain inositol in patients with chronic liver disease may increase their sensitivity of serum sodium levels fluctuations [22].

For one thing, among the 26 cases reported in collected literature, 12 cases (84.6\%) appeared an excessive correction of perioperative hyponatremia, 10 cases (38.5\%) appeared preoperative hepatic encephalopathy, and 9 cases showed both (34.6\%). The time for the symptom appearance of those who had a history of correcting hyponatremia too quickly was $8.45 \pm 5.20$ days, while the others' was $11.50 \pm 5.20$ days, and statistically significant difference existed between the two groups $(P<0.05)$. Meanwhile, the time for the symptom appearance of those who had a history of preoperative hepatic encephalopathy was $6.90 \pm 4.30$ days, while the others' was $10.19 \pm 5.36$ days, and statistically significant difference also existed between the two groups $(P<0.05)$. For another, cases 1, 2, 3 in the 4 cases of our hospital all showed a great fluctuation of serum sodium levels ( $>12$ $\mathrm{mmol} / \mathrm{L}$ in 24 hours) (Table 3), 3 of which existed a preoperative hyponatremia. Only 1 case was diagnosed as preoperative hepatic encephalopathy, and all had hepatic encephalopathy after the liver transplantation. It is suggested that perioperative hepatic encephalopathy may have a close relationship with CPM after liver transplantation.

\section{Immunosuppressive Agents}

CsA and FK506, the two major immunosuppressive agents given to cases after liver transplantation, are both calcineurin inhibitors and play an immunosuppressive effect by combined with immunophilin [23]. However, they may have neurotoxic effects with a high serum concentration. Studies also found that CsA [7] [19] [24] and FK506 have a positive correlation with the occurrence of CPM [24]. Researchers found that CsA and FK506 can damage the intracranial vascular bed, which may subsequently cause vasospasm and microvascular injury [25], and this intracranial vascular injury may be more serious when accompanied by an internal environment disorder. Drug's metabolism and clearance are very slow in liver transplant patients who have impaired liver and renal functions. Therefore, CsA or FK506 might show a neurotoxic effect on these patients even within therapeutic doses [26]. In addition, glucocorticoids can also result in the nervous system injury through its induced immune suppression or a direct toxic effect. Some researchers found that glucocorticoids also have a synergy effect with calcineurin inhibitors, by which they may enlarge their nervous toxic effect. Among the 4 cases of our center, 3 cases had a high FK506 concentration and the left one case had a rapid correction of hyponatremia and multiple organ failure before the operation, all of which may cause the metabolic disorder of the immunosuppressive agents. So it is rational to consider the electrolyte fluctuations during surgery and elevating speed of plasma concentration of immunosuppressive agents as the risk factors for CPM.

\section{Clinical Manifestation and Diagnosis}

The clinical manifestation of CPM after liver transplantation is complex and non-specific, and it generally occurs in 2 - $11 \mathrm{~d}$ after the operation with an average time of $7 \mathrm{~d}$ [22]. In severe cases, it is manifested as spastic paralysis, pseudobulbar palsy, ataxia, locked-in syndrome and so on (Figure 2), and some cases were often misdiagnosed as mental illness for only some psychiatric symptoms. In addition, some concomitant factors, such as hypoxia, hepatic encephalopathy, electrolyte and metabolic disorders, infection etc., can result in coma and psychological symptoms before surgery and subsequently bring some interference for timely diagnosis of CPM. Therefore, it is necessary to consider the possibility of CPM after liver transplantation when patients show unexplained mental disorders. Particularly, there could probably be a higher possibility of CPM for the patients with preoperative hepatic encephalopathy who appear psychiatric symptoms and even coma again after regained consciousness after liver transplantation [27]-[30]. At the present, the diagnosis of CPM mainly based on brain 
MRI scanning, but it should be noted that the MRI of some cases would not show obvious performance until 2 3 weeks after the onset of symptoms [31]. Such as case 4 in our hospital, MRI brain scanning was given twice on day 3 and day 17 after the onset of symptoms respectively, but not any significant change was found in the brain parenchyma until on day 23 when the result of MRI scan was positive. Therefore, it may not be appropriate to exclude those suspected cases only by one negative MRI result, and the combination of clinical symptoms and multiple inspections is indispensable.

\section{Prevention and Treatment}

For the little knowledge about the etiology and effective treatment of CPM, its prevention must be the focus. Many risk factors may be involved in inducing CPM after liver transplantation as mentioned above, so it is essential to do as follows for perioperative liver transplant cases: 1) Correct hyponatremia slowly (24 h correction rate $<12 \mathrm{mmol} / \mathrm{L}$ ), and pay attention to other electrolyte disorders for maintaining the plasma osmolality at normal range; 2) Prevent the neurotoxicity of the immunosuppressant by adjusting the dosage of CsA or FK506 appropriately according to the serum drug concentration; 3 ) Protect the nervous system function before surgery as soon as possible and avoid other incentives of hepatic encephalopathy.

As for the treatment, as the presence of spastic paralysis, varying degrees of conscious disturbance and weakened cough reflex, CPM patients often suffer co-infection, renal failure and other complications which can result in the internal environment disorder, important organs dysfunction and poor prognosis [2], so it is necessary to pay special attention to prevention and treatment of complication, such as aspiration pneumonia infection, retrograde urinary tract infection, renal failure and so on. 2 cases of our center co-suffered respiratory tract infection and1 of them recovered after anti-infective treatment. 1 case of our hospital suffered acute renal failure and was given the continuous renal replacement therapy. Animal experiments and clinical reports imply that timely and reasonable application of glucocorticoid has a certain effect on the prevention and treatment of CPM [32] [33], so we gave large doses of glucocorticoids to 4 cases of our hospital and obtained a certain effect. Besides, nutrition and long-term exercise of neurological function was also given for the treatment of the 4 cases of our hospital. Furthermore, some case reports implied that plasmapheresis, immune globulin, thyrotropin-releasing hormone and hyperbaric oxygen therapy may be effective to CPM cases [34]-[36], but the exact effect requires further clinical validation.

\section{Prognosis}

The prognosis of CPM after liver transplant is still uncertain. Some scholars believe that the CPM is a self-limited disease [37] and its prognosis is good. Meanwhile, some others deem that the prognosis of CPM after liver transplantation is ominous [3] for its high mortality (one of the major causes of death after liver transplantation). All of the 4casesof our hospital were cured, but were still left over sequel to varied degrees. However, only 11 cases (42.3\%) among the 26 cases reported in the literature survived and the other 15 cases (57.7\%) died.CPM after liver transplantation often occurs in the high-incidence period of the intra-abdominal hemorrhage, vascular thrombosis, and graft dysfunction and other complications of liver transplantation. Moreover, CPM can cause lung infections and other complications itself, so prevention of other complications is very important. According to the analysis above, we believe that the prognosis of CPM after liver transplantation is still not optimistic at present, and prevention measures for the incentive and symptomatic treatment is crucial.

In addition, it is widely believed that preoperative hepatic encephalopathy is not only an important incentive of CPM, but also has a close relationship with its prognosis. But in a total of 30 cases in our hospital and reported in the literature, 6 cases (54.5\%) of 11 cases which suffered preoperative hepatic encephalopathy died, and 9 cases (36.8\%) of 19 cases without preoperative hepatic encephalopathy died, showing no significant difference between the two groups. There maybe two reasons for this result. For one thing, the number of cases we collected was not big enough so that it could not represent the overall situation of CPM after liver transplantation. For another, subjective differences in the diagnostic criteria of hepatic encephalopathy among different liver transplantation centers may affect the accuracy of the comprehensive analysis. Therefore, it is necessary to make a more objective and effective diagnostic criteria.

\section{Conclusion}

As a seldom-happened complication, the etiology and pathogenesis of CPM after liver transplantation are not 
understood completely, and its diagnosis mainly relies on brain MRI presently. For the high mortality and nonspecific treatment, both the prevention of the probable incentive and appropriate diagnosis and treatment are of great importance for improving the prognosis of CPM after liver transplantation.

\section{References}

[1] Yang, X.H., Zhang, J.W. and Qi, B.K. (2003) Retrospective Analysis of Central Pontine Myelinolysis. Journal of Qiqihar Medical College, 24, 742-743.

[2] Yu, J., Zheng, S., Liang, T.B., et al. (2004) Possible Causes of Central Pontine Myelinolysis after Liver Transplantation. World Journal of Gastroenterology, 10, 2540-2543.

[3] Liang, T.B., Ke, Q.H., Zheng, S.S., et al. (2005) Central Pontine Myelinolysis after Liver Transplantation: Report of Three Cases. Chinese Journal of Organ Transplantation, 26, 292-293.

[4] Wu, J.W. (2005) MRI Findings after Liver Transplantation the Pons Central Myelinolysis. Chinese Journal of Medical Imaging, 13, 452-454.

[5] Cheng, Y. and Li, C.Y. (2006) One Cases of the Postoperative Central Pontine Myelinolysis Liver Transplant. Stroke and Nervous Diseases, 13, 186-186.

[6] Guo, Y., Sun, Wei, Q., B.A., et al. (2007) MRI Findings after Liver Transplant Center of the Pontine Myelinolysis. Chinese Journal of Medical Imaging, 15, 389-391.

[7] Cai, C.J., Lu, M.Q., An, Y.L., et al. (2007) Diagnosis and Treatment of Postoperative Liver Transplantation Pons Central Myelinolysis Disease. Journal of Southern Medical University, 27, 849-851.

[8] Hui, B. (2008) Diagnosis and Treatment of Central Pontine Myelinolysis after Liver Transplantation: A Report of Five cases. Shandong University, Jinan.

[9] Ma, Y., Tai Q., He, X.-S., et al. (2009) Prophylaxis and Management of Neuropsychiatric Complications after Liver Transplantation. Chinese Journal of Practical Surgery, 29.

[10] Zhang, H.B., Zhang, M.Y. and Cao Y.Y. (2009) Cause Analysis for Central Pontine Myelinolysis after Liver Transplantation. The Journal of Clinical Anesthesiology, 25.

[11] Zhang, S.F., Cui, L.L., Yan, S.M., et al. (2009) Central Pontine Myelinolysis after Liver Transplantation: 5 Cases Report. Chinese Journal of Difficult and Complicated Cases, 8, 587-590.

[12] Zhang, Z.W., Kang, Y., Deng, L.J., et al. (2009) Therapy of Central Pontine Myelinolysis Following Living Donor Liver Transplantation: Report of Three Cases. World Journal of Gastroenterology, 15, 3960. http://dx.doi.org/10.3748/wjg.15.3960

[13] Mu, X.T. and Wang, H. (2009) MR and DWI Imaging of Central Pontine Myelinolysis after Liver Transplantation. Chinese Medical Equipment Journal, 30, 73-73.

[14] Yi, S.H., Li, M.R., Yi, H.M., et al. (2010) Central Pontine Myelinolysis Following Liver Transplantation: Five Cases Report. Organ Transplantation, 1, 77-80.

[15] Sun, L.Y., Xu, W.T., Zhu, Z.J., et al. (2010) Cases Analysis of Our Cases of Central Pontine Myelinolysis after Liver Transplantation. Tianjin Medical Journal, 5, 044.

[16] Bonham, C.A., Dominguez, E.A., Fukui, M.B., et al. (1998) Central Nervous System Lesions in Liver Transplant Recipients: Prospective Assessment of Indications for Biopsy and Implications for Management. Transplantation, 66, 1596-1604. http://dx.doi.org/10.1097/00007890-199812270-00005

[17] Martin, R.J. (2004) Central Pontine and Extrapontine Myelinolysis: The Osmotic Demyelination Syndromes. Journal of Neurology, Neurosurgery \& Psychiatry, 75, iii22-iii28. http://dx.doi.org/10.1136/jnnp.2004.045906

[18] Laureno, R. (2004) Central Pontine Myelinolysis Following Rapid Correction of Hyponatremia. Annals of Neurology, 13, 232-242. http://dx.doi.org/10.1002/ana.410130303

[19] Jun, Y., Liang, T.-B. and Zheng, S.-S. (2004) Progress in Etiology, Diagnosis and Treatment of Central Pontine Myelinolysis after Liver Transplantation. National Medical Journal of China, 84, 1052-1054.

[20] Luo, Y.-Q., Guo W.-Y. and Fu Z.-R. (2010) Demyelinative Diseases after Liver Transplantation: The Etiology. Academic Journal of Second Military Medical University, 1, 104-107.

[21] Abbasoglu, O., Goldstein, R., Vodapally, M., et al. (1998) Liver Transplantation in Hyponatremic Cases with Emphasis on Central Pontine Myelinolysis. Clinical Transplantation, 12, 263.

[22] Bronster, D.J., Emre, S., Boccagni, P., et al. (2001) Central Nervous System Complications in Liver Transplant Recipients-Incidence, Timing, and Long-Term Follow-Up. Clinical Transplantation, 14, 1-7. http://dx.doi.org/10.1034/j.1399-0012.2000.140101.x 
[23] Shi, Y.H., Fan, J., Wu Z.Q., et al. (2004) Retrospective Analysis of Neuropsychiatric Complications of Liver Transplantation. Journal of Surgery Concepts \& Practice, 8, 451-453.

[24] Schwartz, R., Bravo, S., Klufas, R., et al. (1995) Cyclosporine Neurotoxicity and Its Relationship to Hypertensive Encephalopathy: CT and MR Findings in 16 Cases. American Journal of Roentgenology, 165, 627-631. http://dx.doi.org/10.2214/ajr.165.3.7645483

[25] Bekersky, I., Dressler, D. and Mekki, Q. (2001) Effect of Time of Meal Consumption on Bioavailability of a Single Oral 5 mg Tacrolimus Dose. The Journal of Clinical Pharmacology, 41, 289-297. http://dx.doi.org/10.1177/00912700122010104

[26] Yuan, C.H. and Liu, Y.F. (2004) Central Nervous System Toxicity Induced by Tacrolimus after Liver or Renal Transplantation. Journal of Chinese Clinical Medicine, 5, 10-11.

[27] Yi, S.H., Li, H., Yang, Y., et al. (2007) Major Neurological Complications Following Liver Transplantation and Their Management. Journal of Southern Medical University, 27, 1310-1313.

[28] Saner, F.H., Koeppen, S., Meyer, M., et al. (2008) Treatment of Central Pontine Myelinolysis with Plasmapheresis and Immunoglobulins in Liver Transplant Patient. Transplant International, 21, 390-391. http://dx.doi.org/10.1111/j.1432-2277.2007.00608.x

[29] Ramia, J.M., Villar, J., Castaño, J., et al. (2006) Mielinólisis Central Pontina tras Trasplante Hepático. Cirugía Española, 80, 411-412. http://dx.doi.org/10.1016/S0009-739X(06)70999-X

[30] Kim, B.S., Lee, S.G., Hwang, S., et al. (2007) Neurologic Complications in Adult Living Donor Liver Transplant Recipients. Clinical transplantation, 21, 544-547. http://dx.doi.org/10.1111/j.1399-0012.2007.00687.x

[31] Yuan, H. and Qi, J. (2008) Progress in MRI Diagnosis of the Center of the Pontine Myelinolysis. Shandong Medical Journal, 48, 107-108.

[32] Murase, T., Sugimura, Y., Takefuji, S., et al. (2006) Mechanisms and Therapy of Osmotic Demyelination. The American Journal of Medicine, 119, S69-S73. http://dx.doi.org/10.1016/j.amjmed.2006.05.010

[33] Ellison, G.W. and Myers, LW. (1980) Immunosuppressive Drugs in Multiple Sclerosis Pro and Con. Neurology, 30, 28-32. http://dx.doi.org/10.1212/WNL.30.7 Part 2.28

[34] Yoon, K., Fong, K., Koh, D., et al. (2000) Central Pontine Myelinolysis a Rare Manifestation of CNS Sjogren’s Syndrome. Lupus, 9, 471-473. http://dx.doi.org/10.1191/096120300678828550

[35] Chemaly, R., Halaby, G., Mohasseb, G., et al. (1998) Extrapontine Myelinolysis: Treatment with TRH. Revue Neurologique, 154, 163-165.

[36] Zhang, L.-J., Huang, X.-N., Wang, M. and Zhang, J.-J. (1995) A Case of Hyperbaric Oxygen Treatment of Central Pontine Myelinolysis. Chinese Journal of Nautical Medicine and Hyperbaric Medicine, 270, 333-313.

[37] Liu, Y., Dong, W.W., Liu, Y., et al. (2001) Central Pontine Myelinolysis: A Report of 5 Cases with Autopsy Pathological Analysis. Chinese Journal of Neurology, 34, 326-328. 